\title{
TYPES ET CONTRAGRÉDIENTES
}

\author{
par \\ Guy Henniart \& Vincent Sécherre
}

\begin{abstract}
Résumé. - Soit $\mathrm{G}$ un groupe réductif $p$-adique, et soit $\mathrm{R}$ un corps algébriquement clos. Soit $\pi$ une représentation lisse de $\mathrm{G}$ dans un espace vectoriel V sur R. Fixons un sous-groupe ouvert et compact $\mathrm{K}$ de $\mathrm{G}$ et une représentation lisse irréductible $\varrho$ de $\mathrm{K}$ dans un espace vectoriel $\mathrm{W}$ de dimension finie sur R. Sur l'espace $\operatorname{Hom}_{K}(\mathrm{~W}, \mathrm{~V})$ agit l'algèbre d'entrelacement $\mathcal{H}(\mathrm{G}, \mathrm{K}, \mathrm{W})$. Nous examinons la compatibilité de ces constructions avec le passage aux représentations contragrédientes $\mathrm{V}^{\vee}$ et $\mathrm{W}^{\vee}$, et donnons en particulier des conditions sur $\mathrm{W}$ ou sur la caractéristique de $\mathrm{R}$ pour que le comportement soit semblable au cas des représentations complexes. Nous prenons un point de vue abstrait, n'utilisant que des propriétés générales de G. Nous terminons par une application à la théorie des types pour le groupe $\mathrm{GL}_{n}$ et ses formes intérieures sur un corps local non archimédien.
\end{abstract}

\begin{abstract}
Let $\mathrm{G}$ be a $p$-adic reductive group, and $\mathrm{R}$ an algebraically closed field. Let $\pi$ be a smooth representation of $G$ on an R-vector space V. Fix an open compact subgroup $\mathrm{K}$ of $\mathrm{G}$ and a smooth irreducible representation of $\mathrm{K}$ on a finite-dimensional $\mathrm{R}$-vector space $\mathrm{W}$. The space $\operatorname{Hom}_{\mathrm{K}}(\mathrm{W}, \mathrm{V})$ is a right module over the intertwining algebra $\mathcal{H}(\mathrm{G}, \mathrm{K}, \mathrm{W})$. We examine how those constructions behave when we pass to the contragredient representations $\mathrm{V}^{\vee}$ and $\mathrm{W}^{\vee}$, and we give conditions under which the behaviour is the same as in the case of complex representations. We take an abstract viewpoint and use only general properties of G. In the last section, we apply this to the theory of types for the group $\mathrm{GL}_{n}$ and its inner forms over a non-Archimedean local field.
\end{abstract}

2010 Mathematics Subject Classification: 22E50

Keywords and Phrases: Modular representations of $p$-adic reductive groups, Types, Contragredient, Intertwining

\section{$\S 1$. Introduction}

1.1. - Soient $p$ un nombre premier et $\mathrm{G}$ un groupe réductif $p$-adique. Dans l'étude des représentations lisses de $\mathrm{G}$ dans des espaces vectoriels complexes, la restriction aux sous-groupes ouverts et compacts joue un rôle important $[3,4]$. Dans ce contexte, on fixe un sous-groupe ouvert et compact $\mathrm{K}$ de $\mathrm{G}$ et une représentation lisse irréductible $\varrho$ de $\mathrm{K}$ dans un espace vectoriel complexe $\mathrm{W}$, de dimension finie. Pour toute représentation lisse $\pi$ de $\mathrm{G}$ dans un espace vectoriel complexe $\mathrm{V}$, on forme l'espace d'entrelacement $\operatorname{Hom}_{\mathrm{K}}(\mathrm{W}, \mathrm{V})$ qui est naturellement un module à droite sur l'algèbre d'entrelacement - ou algèbre de Hecke - $\mathcal{H}(\mathrm{G}, \mathrm{K}, \mathrm{W})$. Passant aux contragrédientes, l'espace $\operatorname{Hom}_{K}\left(\mathrm{~W}^{\vee}, \mathrm{V}^{\vee}\right)$ est naturellement un module à droite sur $\mathcal{H}\left(\mathrm{G}, \mathrm{K}, \mathrm{W}^{\vee}\right)$. Mais cette dernière algèbre est naturellement anti-isomorphe à $\mathcal{H}(\mathrm{G}, \mathrm{K}, \mathrm{W})$. Dans ce cas classique, les représentations lisses de $K$ sont semi-simples, et $\operatorname{Hom}_{K}\left(W^{\vee}, V^{\vee}\right)$ s'identifie au dual 
de $\operatorname{Hom}_{\mathrm{K}}(\mathrm{W}, \mathrm{V})$, comme module à gauche sur $\mathcal{H}(\mathrm{G}, \mathrm{K}, \mathrm{W})$ : nous retrouverons plus loin ce cas particulier.

1.2. - L'arithmétique des formes modulaires a imposé l'étude des représentations lisses de G dans des espaces vectoriels sur un corps algébriquement clos $\mathrm{R}$, de caractéristique quelconque $[\mathbf{9}, \mathbf{7}, \mathbf{5}]$. En l'absence de méthodes analytiques, la restriction aux sous-groupes ouverts et compacts est en ce cas un outil encore plus important que dans le cas complexe. On a de la même façon que plus haut des modules d'entrelacement $\operatorname{Hom}_{K}(\mathrm{~W}, \mathrm{~V})$ sur des algèbres de Hecke $\mathcal{H}(\mathrm{G}, \mathrm{K}, \mathrm{W})$ à coefficients dans $\mathrm{R}$.

Dans le cas où la caractéristique de $\mathrm{R}$ n'est pas $p$, on dispose d'une bonne théorie des contragrédientes, et il est nécessaire d'examiner le comportement des modules $\operatorname{Hom}_{\mathrm{K}}(\mathrm{W}, \mathrm{V})$ par passage aux contragrédientes.

Comme dans le cas complexe, on dispose d'un anti-isomorphisme d'algèbres de $\mathcal{H}(\mathrm{G}, \mathrm{K}, \mathrm{W})$ sur $\mathcal{H}\left(\mathrm{G}, \mathrm{K}, \mathrm{W}^{\vee}\right)$, de sorte que $\operatorname{Hom}_{\mathrm{K}}\left(\mathrm{W}^{\vee}, \mathrm{V}^{\vee}\right)$ est un module à gauche sur $\mathcal{H}(\mathrm{G}, \mathrm{K}, \mathrm{W})$. Par

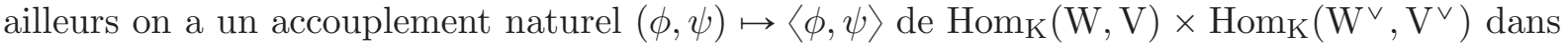
R. Cependant cet accouplement peut être dégénéré. On cherche dans quelles conditions il est non dégénéré, et hermitien au sens où $\langle\phi \mathrm{T}, \psi\rangle=\langle\phi, \mathrm{T} \psi\rangle$ pour $\mathrm{T} \in \mathcal{H}(\mathrm{G}, \mathrm{K}, \mathrm{W})$. En particulier, nous obtenons le résultat suivant (voir la remarque 2.4 et le théorème 3.4 ).

Proposition 1.1. - Supposons que le pro-ordre de K est inversible dans R. Alors l'accouplement $\operatorname{Hom}_{\mathrm{K}}(\mathrm{W}, \mathrm{V}) \times \operatorname{Hom}_{\mathrm{K}}\left(\mathrm{W}^{\vee}, \mathrm{V}^{\vee}\right) \rightarrow \mathrm{R}$ est non dégénéré et hermitien.

1.3. - Supposons $\mathrm{R}$ de caractéristique $\ell$ différente de $p$, et examinons le cas, inévitable en pratique, où $\ell$ divise le pro-ordre de $\mathrm{K}$. Alors les représentations lisses de $\mathrm{K}$ ne sont plus forcément semi-simples, et l'accouplement peut effectivement être dégénéré. Cependant une situation fréquente est celle où $K$ possède un sous-groupe distingué fermé $K_{1}$, de pro-ordre premier à $\ell$, qui vérifie les deux conditions suivantes :

(1) La restriction de $\varrho$ à $\mathrm{K}_{1}$ est multiple d'une représentation irréductible $\eta$.

(2) Le composant isotypique de $\pi$ de type $\varrho$ est aussi le composant isotypique de type $\eta$.

En ce cas, l'accouplement plus haut est encore non dégénéré (voir ci-dessous §2.4), et si de plus $\ell$ ne divise pas la dimension de $\mathrm{W}$, il est aussi hermitien (théorème 3.4).

1.4. - Le but de cette courte note est d'étudier la situation générale. Nous prenons un point de vue abstrait, où $\mathrm{G}$ est un groupe localement profini et $\mathrm{R}$ un corps commutatif quelconque. Dans un premier temps, nous examinons les représentations lisses de $\mathrm{K}$ dans des espaces vectoriels sur $\mathrm{R}$, et leur comportement par passage à la contragrédiente. Nous passons ensuite à l'étude des modules d'entrelacement. Notre conclusion essentielle réside dans les formules (3.5) et (3.6) du $\S 3.2$, dont la comparaison permet ensuite de donner des critères et exemples où l'accouplement est hermitien.

1.5. - Pour finir, nous donnons des applications de ce travail à la théorie des types d'un groupe linéaire général sur un corps local non archimédien F. Nous prouvons que les types simples de Bushnell-Kutzko - et plus généralement leur version modulaire construite dans [6] pour les formes intérieures de $\mathrm{GL}_{n}(\mathrm{~F}), n \geqslant 1$ - satisfont aux conditions requises pour que l'accouplement soit non dégénéré et hermitien, et ce pour toute représentation $\mathrm{V}$ de $\mathrm{G}$ qui est un sous-quotient d'une représentation engendrée par son composant isotypique de type $\varrho$ (théorème 4.1). 


\section{§2. Représentations de $\mathrm{K}$}

2.1. - On fixe désormais un corps commutatif $\mathrm{R}$, et on note $\ell$ sa caractéristique. Par représentation $(\pi, \mathrm{V})$ d'un groupe $\mathrm{G}$, on entendra représentation dans un espace vectoriel $\mathrm{V}$ sur le corps R.

Dans ce paragraphe 2, on fixe un groupe profini K. Si $(\pi, \mathrm{V})$ est une représentation lisse de K, sa contragrédiente $\pi^{\vee}$ est la représentation naturelle de $\mathrm{K}$ dans le sous-espace $\mathrm{V}^{\vee}$ du dual $\mathrm{V}^{*}$ de $\mathrm{V}$ formé des formes linéaires sur $\mathrm{V}$ dont le stabilisateur dans $\mathrm{K}$ est ouvert. L'homomorphisme d'évaluation $\mathrm{V} \otimes \mathrm{V}^{\vee} \rightarrow \mathrm{R}$ défini par $(x, \lambda) \mapsto \lambda(x)$ induit un entrelacement naturel de $\mathrm{V}$ vers son bicontragrédient $\mathrm{V}^{\vee \vee}$.

Si $\mathrm{V}$ est de dimension finie, $\pi$ se factorise par un quotient fini de $\mathrm{K}$, de sorte que $\mathrm{V}$ et $\mathrm{V}^{\vee}$ ont même dimension et que l'entrelacement naturel de $\mathrm{V}$ dans $\mathrm{V}^{\vee} \vee$ est un isomorphisme. Montrons par un exemple que ces bonnes propriétés ne s'étendent pas forcément aux représentations de dimension infinie.

Exemple 2.1. - On suppose que $\ell$ n'est pas nul, et aussi que le pro-ordre de $\mathrm{K}$ est divisible par $\ell^{\infty}$; autrement dit, pour chaque entier $r \geqslant 1, \mathrm{~K}$ possède un sous-groupe ouvert, qu'on peut prendre distingué, d'indice divisible par $\ell^{r}$. Considérons la représentation (lisse) de $\mathrm{K}$ dans l'espace $\mathrm{V}$ des fonctions localement constantes de $\mathrm{K}$ dans $\mathrm{R}$, disons par translations à droite. Prouvons que $\mathrm{V}^{\vee}$ est nul.

Soit $\lambda \in \mathrm{V}^{\vee}$. Il suffit de prouver que, si J est un sous-groupe ouvert de $\mathrm{K}$ fixant $\lambda$, alors $\lambda$ est nulle sur le sous-espace vectoriel de $\mathrm{V}$ formé des fonctions invariantes à droite par J. Grâce à l'hypothèse, il existe un sous-groupe ouvert distingué $\mathrm{J}^{\prime}$ de $\mathrm{J}$ dont l'indice est divisible par $\ell$. Fixons un système $\mathrm{X}$ de représentants de $\mathrm{J}^{\prime} \backslash \mathrm{J}$ dans $\mathrm{J}$. Pour $g$ dans $\mathrm{K}$, la fonction caractéristique de $g \mathrm{~J}$ est somme des fonctions caractéristiques $\chi_{k}$ de $g \mathrm{~J} k$, pour $k$ parcourant $\mathrm{X}$; mais $\lambda\left(\chi_{k}\right)$ ne dépend pas de $k$, et le cardinal de $\mathrm{X}$ est divisible par $\ell$, de sorte que $\lambda$ s'annule sur la fonction caractéristique de $g \mathrm{~J}$.

2.2. - Fixons une représentation lisse absolument irréductible $(\varrho, \mathrm{W})$ de $\mathrm{K}$ : elle est donc de dimension finie sur R. Soit $(\pi, \mathrm{V})$ une représentation lisse de K. La donnée de deux entrelacements $\phi \in \operatorname{Hom}_{K}(\mathrm{~W}, \mathrm{~V})$ et $\psi \in \operatorname{Hom}_{\mathrm{K}}\left(\mathrm{W}^{\vee}, \mathrm{V}^{\vee}\right)$ définit un entrelacement :

$$
\phi \otimes \psi \in \mathrm{Hom}_{\mathrm{K} \times \mathrm{K}}\left(\mathrm{W} \otimes \mathrm{W}^{\vee}, \mathrm{V} \otimes \mathrm{V}^{\vee}\right) .
$$

Composant avec l'évaluation $\mathrm{V} \otimes \mathrm{V}^{\vee} \rightarrow \mathrm{R}$, on obtient un entrelacement dans $\mathrm{Hom}_{\mathrm{K}}\left(\mathrm{W} \otimes \mathrm{W}^{\vee}, \mathrm{R}\right)$, où $\mathrm{K}$ agit sur $\mathrm{W} \otimes \mathrm{W}^{\vee}$ par l'action diagonale. Comme $\varrho$ est absolument irréductible de dimension finie, l'espace $\operatorname{Hom}_{K}\left(\mathrm{~W} \otimes \mathrm{W}^{\vee}, \mathrm{R}\right)$ est de dimension 1 , de base l'évaluation $\mathrm{W} \otimes \mathrm{W}^{\vee} \rightarrow \mathrm{R}$. On

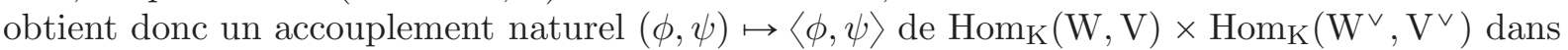
$\mathrm{R}$, donné par la formule :

$$
\psi\left(w^{\vee}\right)(\phi(w))=\langle\phi, \psi\rangle \cdot w^{\vee}(w)
$$

pour $w \in \mathrm{W}$ et $w^{\vee} \in \mathrm{W}^{\vee}$.

Cet accouplement peut être dégénéré, puisque nous avons vu que $\mathrm{V}^{\vee}$ peut être nul sans que V le soit. Étudions cela plus avant.

Remarque 2.2. - Comme $\mathrm{W}$ est de dimension finie, le passage au transposé donne un isomorphisme de $\operatorname{Hom}_{K}(V, W)$ sur $\operatorname{Hom}_{K}\left(W^{\vee}, V^{\vee}\right)$. De plus, W étant absolument irréductible, la Ralgèbre $\operatorname{End}_{\mathrm{K}}(\mathrm{W})$ est réduite aux homothéties $\mathrm{R} \cdot \mathrm{id} \mathrm{W}_{\mathrm{W}}$. En ces termes, l'accouplement précédent se traduit en la composition $\operatorname{Hom}_{\mathrm{K}}(\mathrm{W}, \mathrm{V}) \times \operatorname{Hom}_{\mathrm{K}}(\mathrm{V}, \mathrm{W}) \rightarrow \operatorname{End}_{\mathrm{K}}(\mathrm{W}) \simeq \mathrm{R}$. 
2.3. - Notons $\mathrm{V}(\varrho)$ la plus grande sous-représentation de V isotypique de type $\varrho$. C'est l'image de $\operatorname{Hom}_{K}(\mathrm{~W}, \mathrm{~V}) \otimes \mathrm{W}$ dans $\mathrm{V}$ par l'application injective $\phi \otimes w \mapsto \phi(w)$. En particulier, si V est isotypique de type $\varrho$, alors $\mathrm{V}^{*}$ est isomorphe à $\operatorname{Hom}_{\mathrm{K}}(\mathrm{W}, \mathrm{V})^{*} \otimes \mathrm{W}^{\vee}$, de sorte que $\mathrm{V}^{\vee}$ est égale à $\mathrm{V}^{*}$ et est isotypique de type $\varrho^{\vee}$. De plus, l'application $\mathrm{V} \rightarrow \mathrm{V}^{\vee}$ est injective. En général, notons $\mathrm{V}_{\varrho}$ le plus grand quotient de $\mathrm{V}$ isotypique de type $\varrho$, et utilisons des notations analogues pour $\mathrm{V}^{\vee}, \varrho^{\vee}$.

Les entrelacements de $\mathrm{V}$ dans $\mathrm{W}$ se factorisent par l'espace $\mathrm{V}_{\varrho}$, ce qui donne un isomorphisme de $\operatorname{Hom}_{K}\left(\mathrm{~V}_{\varrho}, \mathrm{W}\right)$ sur $\operatorname{Hom}_{\mathrm{K}}(\mathrm{V}, \mathrm{W})$ et, par transposition, un isomorphisme de $\operatorname{Hom}_{\mathrm{K}}\left(\mathrm{W}^{\vee},\left(\mathrm{V}_{\varrho}\right)^{\vee}\right)$ sur $\operatorname{Hom}_{K}\left(\mathrm{~W}^{\vee}, \mathrm{V}^{\vee}\right)$ (voir la remarque 2.2). Considérons alors le diagramme suivant où les flèches verticales proviennent de la projection $\mathrm{V} \rightarrow \mathrm{V}_{\varrho}$, celle de droite étant un isomorphisme :

$$
\begin{array}{r}
\operatorname{Hom}_{\mathrm{K}}(\mathrm{W}, \mathrm{V}) \times \operatorname{Hom}_{\mathrm{K}}\left(\mathrm{W}^{\vee}, \mathrm{V}^{\vee}\right) \rightarrow \mathrm{R} \\
\uparrow \\
\downarrow \\
\operatorname{Hom}_{\mathrm{K}}\left(\mathrm{W}, \mathrm{V}_{\varrho}\right) \times \operatorname{Hom}_{\mathrm{K}}\left(\mathrm{W}^{\vee},\left(\mathrm{V}_{\varrho}\right)^{\vee}\right) \rightarrow \mathrm{R}
\end{array}
$$

Comme $\mathrm{V}_{\varrho}$ est isotypique de type $\varrho$, l'accouplement de la ligne du bas est non dégénéré : plus précisément, son noyau à droite et son noyau à gauche sont nuls, et il induit un isomorphisme de $\operatorname{Hom}_{\mathrm{K}}\left(\mathrm{W}^{\vee},\left(\mathrm{V}_{\varrho}\right)^{\vee}\right)$ sur $\operatorname{Hom}_{\mathrm{K}}\left(\mathrm{W}, \mathrm{V}_{\varrho}\right)^{*}$; si de plus $\mathrm{V}_{\varrho}$ est de dimension finie, il induit un isomorphisme de $\operatorname{Hom}_{\mathrm{K}}\left(\mathrm{W}, \mathrm{V}_{\varrho}\right)$ sur $\operatorname{Hom}_{\mathrm{K}}\left(\mathrm{W}^{\vee},\left(\mathrm{V}_{\varrho}\right)^{\vee}\right)^{*}$. Remarquant que le diagramme est compatible aux accouplements et que les entrelacements de $\mathrm{W}$ dans $\mathrm{V}$ prennent leurs valeurs dans $\mathrm{V}(\varrho)$, on a obtenu le résultat suivant.

Proposition 2.3. - Supposons que l'application $\mathrm{V}(\varrho) \rightarrow \mathrm{V}_{\varrho}$ est un isomorphisme. L'accouplement :

$$
\operatorname{Hom}_{\mathrm{K}}(\mathrm{W}, \mathrm{V}) \times \operatorname{Hom}_{\mathrm{K}}\left(\mathrm{W}^{\vee}, \mathrm{V}^{\vee}\right) \rightarrow \mathrm{R}
$$

induit alors d'une part un isomorphisme de $\operatorname{Hom}_{\mathrm{K}}\left(\mathrm{W}^{\vee}, \mathrm{V}^{\vee}\right)$ sur $\operatorname{Hom}_{\mathrm{K}}(\mathrm{W}, \mathrm{V})^{*}$, d'autre part une injection de $\operatorname{Hom}_{\mathrm{K}}(\mathrm{W}, \mathrm{V})$ dans $\operatorname{Hom}_{\mathrm{K}}\left(\mathrm{W}^{\vee}, \mathrm{V}^{\vee}\right)^{*}$, qui est une bijection si $\mathrm{V}(\varrho)$ est de dimension finie.

Remarque 2.4. - Si le pro-ordre de $\mathrm{K}$ est inversible dans $\mathrm{R}$, toute représentation lisse de $\mathrm{K}$ est semi-simple et, quel que soit V, l'hypothèse de la proposition est satisfaite. C'est le cas en particulier si $\mathrm{R}$ est de caractéristique nulle, par exemple si $\mathrm{R}=\mathbb{C}$.

2.4. - Dans la pratique, le pro-ordre de K n'est pas forcément inversible dans R. Mais il arrive (voir [6]) que $\mathrm{K}$ possède un sous-groupe ouvert distingué $\mathrm{K}_{1}$ ayant les propriétés suivantes :

(1) Le pro-ordre de $\mathrm{K}_{1}$ est inversible dans $\mathrm{R}$.

(2) La restriction de $\varrho$ à $\mathrm{K}_{1}$ est multiple d'une représentation absolument irréductible $\eta$ de $\mathrm{K}_{1}$.

(3) $\mathrm{V}(\varrho)=\mathrm{V}(\eta)$.

Proposition 2.5. - Sous les conditions précédentes, l'application $\mathrm{V}(\varrho) \rightarrow \mathrm{V}_{\varrho}$ est un isomorphisme.

Démonstration. - Comme $\eta$ est la seule représentation irréductible de $\mathrm{K}_{1}$ intervenant dans $\mathrm{W}$, sa classe d'isomorphisme est invariante par conjugaison par $\mathrm{K}$ et $\mathrm{V}_{\eta}$ est une représentation de $\mathrm{K}$. La projection de $\mathrm{V}(\varrho)=\mathrm{V}(\eta)$ dans $\mathrm{V}_{\eta}$ est K-équivariante et, comme le pro-ordre de $\mathrm{K}_{1}$ est inversible dans $\mathrm{R}$, c'est même un isomorphisme. En particulier $\mathrm{V}_{\eta}$ est isotypique de type $\varrho$ et il s'ensuit que $\mathrm{V}_{\varrho}=\mathrm{V}_{\eta}$. 
2.5. - Revenons à notre situation générale, et donnons des conditions nécessaires et suffisantes pour que $\mathrm{V}(\varrho) \rightarrow \mathrm{V}_{\varrho}$ soit un isomorphisme.

Proposition 2.6. - Les conditions suivantes sont équivalentes :

(1) L'application $\mathrm{V}(\varrho) \rightarrow \mathrm{V}_{\varrho}$ est un isomorphisme.

(2) L'accouplement (2.1) induit un isomorphisme entre $\operatorname{Hom}_{\mathrm{K}}(\mathrm{V}, \mathrm{W})$ et $\mathrm{Hom}_{\mathrm{K}}(\mathrm{W}, \mathrm{V})^{*}$.

(3) $\mathrm{V}$ se décompose sous la forme $\mathrm{V}(\varrho) \oplus \mathrm{V}^{\prime}$ avec $\operatorname{Hom}_{\mathrm{K}}\left(\mathrm{W}, \mathrm{V}^{\prime}\right)=\operatorname{Hom}_{\mathrm{K}}\left(\mathrm{V}^{\prime}, \mathrm{W}\right)=\{0\}$.

Démonstration. - On a déjà prouvé que la condition 1 implique la condition 2 (voir la proposition 2.3 et la remarque 2.2) et on vérifie immédiatement que la condition 3 implique la condition 1. Notons $\mathrm{V}^{\prime}$ le noyau de $\mathrm{V} \rightarrow \mathrm{V}_{\varrho}$. Le noyau à droite de $(2.1)$ est formé des $\psi \in \operatorname{Hom}_{\mathrm{K}}(\mathrm{V}, \mathrm{W})$ qui sont nuls sur $\mathrm{V}(\varrho)+\mathrm{V}^{\prime}$ et son noyau à gauche est formé des $\phi \in \operatorname{Hom}_{\mathrm{K}}(\mathrm{W}, \mathrm{V})$ dont l'image est incluse dans $\mathrm{V}(\varrho) \cap \mathrm{V}^{\prime}$. Si la condition 2 est vérifiée, on obtient donc $\mathrm{V}(\varrho) \cap \mathrm{V}^{\prime}=\{0\}$ et :

$$
\operatorname{Hom}_{\mathrm{K}}\left(\mathrm{V} /\left(\mathrm{V}(\varrho)+\mathrm{V}^{\prime}\right), \mathrm{W}\right)=\{0\} .
$$

Comme le quotient $\mathrm{V} /\left(\mathrm{V}(\varrho)+\mathrm{V}^{\prime}\right)$ est isotypique de type $\varrho$, on en déduit que $\mathrm{V}=\mathrm{V}(\varrho) \oplus \mathrm{V}^{\prime}$. On a aussi $\mathrm{V}^{\prime}(\varrho)=\mathrm{V}(\varrho) \cap \mathrm{V}^{\prime}=\{0\}$ et $\mathrm{V}_{\varrho}^{\prime}=\mathrm{V} /\left(\mathrm{V}(\varrho)+\mathrm{V}^{\prime}\right)=\{0\}$, ce qui termine la preuve.

Définition 2.7. - La représentation $\mathrm{V}$ est dite $\mathrm{W}$-semi-simple si les conditions équivalentes de la proposition 2.6 sont vérifiées.

La propriété de W-semi-simplicité n'est stable ni par passage à un sous-objet, ni par passage à un quotient, comme le montre l'exemple ci-dessous.

Exemple 2.8. - On suppose que $\mathrm{K}$ est le groupe $\mathrm{GL}_{2}(k)$, où $k$ est un corps fini dont le cardinal $q$ est d'ordre 2 dans $\mathrm{R}^{\times}$. On considère la représentation de $\mathrm{K}$, par translations à droite, sur l'espace $\mathrm{E}$ des fonctions de $\mathrm{K}$ dans $\mathrm{R}$ qui sont invariantes à gauche par le sous-groupe des matrices triangulaires supérieures. Elle est indécomposable et de longueur 3 : elle admet une unique suite de composition $\{0\} \subseteq \mathrm{X} \subseteq \mathrm{Y} \subseteq \mathrm{E}$, où $\mathrm{X}$ et $\mathrm{E} / \mathrm{Y}$ sont isomorphes au caractère trivial de $\mathrm{K}$ et où $\mathrm{Y} / \mathrm{X}$ est une représentation irréductible cuspidale de $\mathrm{K}$, notée $(\varrho, \mathrm{W})$. Remarquons que $\mathrm{X}$ est le sous-espace des fonctions constantes.

Le quotient $\mathrm{V}=\mathrm{E} / \mathrm{X}$ est indécomposable et de longueur 2. L'espace $\mathrm{V}(\varrho)$ est non nul, isomorphe à $\varrho$, mais on va voir que $\mathrm{V}_{\varrho}$ est réduit à $\{0\}$. Si ce n'était pas le cas, alors $\varrho^{\vee}$ apparaitrait comme sous-représentation de $\mathrm{V}^{\vee}$. Or $\mathrm{V}^{\vee}$ est indécomposable, et son unique sous-représentation irréductible est le caractère trivial de $\mathrm{K}$.

La représentation $\mathrm{E}$ est $\mathrm{W}$-semi-simple, avec $\mathrm{E}(\varrho)=\{0\}$, mais ni son unique quotient $\mathrm{V}$ de longueur 2 ni son unique sous-représentation $\mathrm{Y}$ de longueur 2 ne le sont.

Ceci nous conduira à introduire dans le paragraphe suivant une propriété plus forte. Signalons toutefois les propriétés suivantes.

Proposition 2.9. - (1) On suppose que l'application $\mathrm{V}(\varrho) \rightarrow \mathrm{V}_{\varrho}$ est surjective. Alors pour tout quotient $\mathrm{Q}$ de $\mathrm{V}$, l'application $\mathrm{Q}(\varrho) \rightarrow \mathrm{Q}_{\varrho}$ est surjective.

(2) On suppose que l'application $\mathrm{V}(\varrho) \rightarrow \mathrm{V}_{\varrho}$ est injective. Alors pour toute sous-représentation $\mathrm{X}$ de $\mathrm{V}$, l'application $\mathrm{X}(\varrho) \rightarrow \mathrm{X}_{\varrho}$ est injective.

Démonstration. - Soit X un sous-espace de V stable par K, et soit Q le quotient de V par X. On prouve d'abord (1). Le quotient :

$$
(\mathrm{V}(\varrho)+\mathrm{X}) / \mathrm{X} \simeq \mathrm{V}(\varrho) /(\mathrm{X} \cap \mathrm{V}(\varrho))
$$


est isotypique de type $\varrho$; c'est donc un sous-espace de $\mathrm{Q}(\varrho)$. La composée de $\mathrm{V} \rightarrow \mathrm{Q}$ et $\mathrm{Q} \rightarrow \mathrm{Q} \varrho$ se factorise par $\mathrm{V} \rightarrow \mathrm{V}_{\varrho}$, c'est-à-dire que $\mathrm{Q}_{\varrho}$ est un quotient de $\mathrm{V}_{\varrho}$. Il suffit donc de prouver que l'application $(\mathrm{V}(\varrho)+\mathrm{X}) / \mathrm{X} \rightarrow \mathrm{V}_{\varrho}$ est surjective. On note $\mathrm{V}^{\prime}$ le noyau de $\mathrm{V} \rightarrow \mathrm{V}_{\varrho}$. Alors par hypothèse faite sur $\mathrm{V}(\varrho) \rightarrow \mathrm{V}_{\varrho}$, on a $\mathrm{V}=\mathrm{V}(\varrho)+\mathrm{V}^{\prime}$, ce qui prouve la surjectivité voulue.

On prouve maintenant (2). On note toujours $\mathrm{V}^{\prime}$ le noyau de $\mathrm{V} \rightarrow \mathrm{V}_{\varrho}$. Le quotient :

$$
\mathrm{X} /\left(\mathrm{X} \cap \mathrm{V}^{\prime}\right) \simeq\left(\mathrm{X}+\mathrm{V}^{\prime}\right) / \mathrm{V}^{\prime}
$$

est isotypique de type $\varrho$; c'est donc un quotient de $\mathrm{X}_{\varrho}$. Par hypothèse faite sur $\mathrm{V}(\varrho) \rightarrow \mathrm{V}_{\varrho}$, on a $\mathrm{V}(\varrho) \cap \mathrm{V}^{\prime}=\{0\}$. Ainsi $\mathrm{X}(\varrho)=\mathrm{X} \cap \mathrm{V}(\varrho)$ se plonge dans $\mathrm{X} /\left(\mathrm{X} \cap \mathrm{V}^{\prime}\right)$, ce dont on déduit que l'application $\mathrm{X}(\varrho) \rightarrow \mathrm{X}_{\varrho}$ est injective.

Remarque 2.10. - Soit $\left(\mathrm{V}_{i}\right)_{i \in \mathrm{I}}$ une famille de représentations de $\mathrm{K}$, et soit $\mathrm{V}$ la somme directe des $\mathrm{V}_{i}, i \in \mathrm{I}$. Les conditions suivantes sont équivalentes :

(1) Pour tout $i \in \mathrm{I}$, l'application $\mathrm{V}_{i}(\varrho) \rightarrow \mathrm{V}_{i, \varrho}$ est surjective (resp. injective).

(2) L'application $\mathrm{V}(\varrho) \rightarrow \mathrm{V}_{\varrho}$ est surjective (resp. injective).

2.6. - Introduisons dans ce paragraphe une propriété plus stable que celle de W-semisimplicité.

Définition 2.11. - Une représentation lisse $\mathrm{V}$ de $\mathrm{K}$ est dite fortement $\mathrm{W}$-semi-simple si elle se décompose sous la forme $\mathrm{V}(\varrho) \oplus \mathrm{V}^{\prime}$, où $\mathrm{V}^{\prime}$ ne possède aucun sous-quotient isomorphe à $\mathrm{W}$.

Bien sûr, une représentation fortement W-semi-simple est W-semi-simple (proposition 2.6).

Proposition 2.12. - Tout sous-quotient d'une représentation fortement $\mathrm{W}$-semi-simple de $\mathrm{K}$ est fortement $\mathrm{W}$-semi-simple.

Démonstration. - Soit E une représentation lisse fortement W-semi-simple de K, qu'on écrit sous la forme $\mathrm{E}(\varrho) \oplus \mathrm{E}^{\prime}$.

Lemme 2.13. - Soit $\mathrm{V}$ une sous-représentation de $\mathrm{E}$. Alors $\mathrm{V}=(\mathrm{V} \cap \mathrm{E}(\varrho)) \oplus\left(\mathrm{V} \cap \mathrm{E}^{\prime}\right)$.

Démonstration. - On note U le quotient de $\mathrm{V}$ par $(\mathrm{V} \cap \mathrm{E}(\varrho)) \oplus\left(\mathrm{V} \cap \mathrm{E}^{\prime}\right)$. On va prouver que U est nul. D'une part, U est un quotient de :

$$
\mathrm{V} /\left(\mathrm{V} \cap \mathrm{E}^{\prime}\right) \simeq\left(\mathrm{V}+\mathrm{E}^{\prime}\right) / \mathrm{E}^{\prime} \subseteq \mathrm{E} / \mathrm{E}^{\prime} \simeq \mathrm{E}(\varrho)
$$

qui est isotypique de type $\varrho$. D'autre part, $\mathrm{U}$ est un quotient de :

$$
\mathrm{V} /(\mathrm{V} \cap \mathrm{E}(\varrho)) \simeq(\mathrm{V}+\mathrm{E}(\varrho)) / \mathrm{E}(\varrho) \subseteq \mathrm{E} / \mathrm{E}(\varrho) \simeq \mathrm{E}^{\prime}
$$

qui ne contient aucun sous-quotient irréductible isomorphe à $\varrho$. On en déduit que U est nul. En particulier $\mathrm{V}$ est fortement W-semi-simple, avec $\mathrm{V}(\varrho)=\mathrm{V} \cap \mathrm{E}(\varrho)$ et $\mathrm{V}^{\prime}=\mathrm{V} \cap \mathrm{E}^{\prime}$.

Soit maintenant $\mathrm{X}$ le quotient de $\mathrm{E}$ par la sous-représentation $\mathrm{V}$. D'après le lemme précédent, on voit que $\mathrm{X}=\mathrm{X}(\varrho) \oplus \mathrm{X}^{\prime}$ avec $\mathrm{X}(\varrho)=\mathrm{E}(\varrho) /(\mathrm{V} \cap \mathrm{E}(\varrho))$ et $\mathrm{X}^{\prime}=\mathrm{E}^{\prime} /\left(\mathrm{V} \cap \mathrm{E}^{\prime}\right)$, ce qui prouve que $\mathrm{X}$ est fortement $\mathrm{W}$-semi-simple.

La propriété de $\mathrm{W}$-semi-simplicité forte est stable par sommes directes arbitraires : si $\left(\mathrm{V}_{i}\right)_{i \in \mathrm{I}}$ est une famille de représentations de $\mathrm{K}$, et si $\mathrm{V}$ est la somme directe des $\mathrm{V}_{i}, i \in \mathrm{I}$, alors $\mathrm{V}$ est fortement $\mathrm{W}$-semi-simple si et seulement si chacune des $\mathrm{V}_{i}, i \in \mathrm{I}$, est fortement $\mathrm{W}$-semi-simple. 
En revanche, cette propriété n'est pas stable par extensions : toute représentation irréductible de $\mathrm{K}$ est fortement $\mathrm{W}$-semi-simple, mais une extension non triviale de $\varrho$ par une représentation irréductible non isomorphe à $\varrho$ (s'il en existe) ne l'est pas.

2.7. — Étudions le comportement de la notion de W-semi-simplicité forte par passage à la contragrédiente.

Pour toute représentation lisse $\mathrm{E}$ de $\mathrm{K}$ et pour toute partie $\mathrm{S}$ de $\mathrm{E}^{\vee}$, on notera $\mathrm{S}^{\circ}$ l'orthogonal de $\mathrm{S}$ dans E, c'est-à-dire l'intersection des noyaux des éléments de S.

Proposition 2.14. - Soit E une représentation lisse fortement $\mathrm{W}$-semi-simple de $\mathrm{K}$. Alors sa contragrédiente $\mathrm{E}^{\vee}$ est fortement $\mathrm{W}^{\vee}$-semi-simple.

Démonstration. - Soit E une représentation fortement W-semi-simple de K qu'on décompose sous la forme $\mathrm{E}(\varrho) \oplus \mathrm{E}^{\prime}$ où $\mathrm{E}^{\prime}$ est une sous-représentation de $\mathrm{E}$ ne possèdant aucun sous-quotient isomorphe à $\mathrm{W}$. Sa contragrédiente $\mathrm{E}^{\vee}$ s'écrit $\mathrm{E}(\varrho)^{*} \oplus \mathrm{E}^{\prime \vee}$ et on va montrer que $\mathrm{E}^{\prime \vee}$ ne possède aucun sous-quotient isomorphe à $\mathrm{W}^{\vee}$. On a le lemme immédiat suivant.

Lemme 2.15. - Soient $\mathrm{T}, \mathrm{U}$ des sous-espaces de $\mathrm{E}^{\vee}$ tels que $\mathrm{T} \subseteq \mathrm{U}$. Alors l'application qui $\grave{a} t \in \mathrm{T}^{\circ}$ associe la forme linéaire $\xi \mapsto \xi(t)$ sur $\mathrm{U}$ induit un homomorphisme injectif de $\mathrm{T}^{\circ} / \mathrm{U}^{\circ}$ dans $(\mathrm{U} / \mathrm{T})^{\vee}$. En outre, si $\mathrm{U}$ est de dimension finie, c'est un isomorphisme.

Supposons qu'il y a des sous-espaces $\mathrm{T} \subseteq \mathrm{U}$ de $\mathrm{E}^{\vee \vee}$ stables par $\mathrm{K}$ et tels que le quotient $\mathrm{U} / \mathrm{T}$ est isomorphe à $W^{\vee}$. Quitte à remplacer $U$ par la sous-représentation de $U$ engendrée par un vecteur qui est dans $U$ mais pas dans $T$, on peut supposer que $U$ est de dimension finie. On a ainsi un isomorphisme $\mathrm{T}^{\circ} / \mathrm{U}^{\circ} \simeq(\mathrm{U} / \mathrm{T})^{\vee}$, ce qui contredit l'hypothèse faite sur $\mathrm{E}^{\prime}$.

La réciproque n'est pas vraie en général : si V est la représentation de l'exemple 2.1, alors sa contragrédiente, qui est nulle, est fortement W-semi-simple pour n'importe quel W, tandis que $\mathrm{V}$ n'est fortement W-semi-simple pour aucun W. On a toutefois le résultat suivant.

Proposition 2.16. - Supposons que $\mathrm{K}$ a un sous-groupe ouvert $\mathrm{K}_{1}$ de pro-ordre inversible dans $\mathrm{R}$. Alors une représentation de $\mathrm{K}$ est fortement $\mathrm{W}$-semi-simple si et seulement si sa contragrédiente est fortement $\mathrm{W}^{\vee}$-semi-simple.

Démonstration. - La proposition 2.14 prouve l'une des implications. Par hypothèse sur K, E se plonge naturellement dans son bicontragrédient $\mathrm{E}^{\vee \vee}$. L'implication réciproque se déduit des propositions 2.12 et 2.14 .

2.8. - Revenons finalement à la notion de W-semi-simplicité et à la façon dont cette propriété se comporte par passage à la contragrédiente.

Proposition 2.17. - Supposons que K a un sous-groupe ouvert de pro-ordre inversible dans $\mathrm{R}$. Alors une représentation de $\mathrm{K}$ est $\mathrm{W}$-semi-simple si et seulement si sa contragrédiente est $\mathrm{W}^{\vee}$-semi-simple.

Démonstration. - Étant donnée une représentation lisse E de K qui n'a W ni pour sous-représentation ni pour quotient, il s'agit de montrer que $W^{\vee}$ n'est ni sous-représentation ni quotient de $E^{\vee}$. Supposons que $E^{\vee}$ a une sous-représentation $U^{\vee}$ isomorphe à $W^{\vee}$. D'après le lemme 2.15 avec $\mathrm{T}=\{0\}$, on obtient un isomorphisme $\mathrm{E} / \mathrm{U}^{\circ} \simeq \mathrm{U}^{\vee}$, ce qui contredit le fait que $\mathrm{E}$ n'a pas de quotient isomorphe à W. Si E est de dimension finie sur R, on peut encore raisonner de façon analogue pour prouver que $\mathrm{E}^{\vee} \mathrm{n}$ 'a pas de quotient isomorphe à $\mathrm{W}^{\vee}$. 
Il reste à prouver que $\mathrm{E}^{\vee} \mathrm{n}^{\prime} \mathrm{a}$ pas de quotient isomorphe à $\mathrm{W}^{\vee}$ dans le cas où $\mathrm{E}$ n'est pas de dimension finie sur R. Puisque $\mathrm{W}$ est lisse et de dimension finie, on peut supposer que $\mathrm{K}$ possède un sous-groupe ouvert distingué $\mathrm{K}_{1}$ de pro-ordre inversible dans $\mathrm{R}$, et que $\mathrm{K}_{1}$ agit trivialement sur W. Écrivons $\mathrm{E}=\mathrm{E}^{\mathrm{K}_{1}} \oplus \mathrm{E}_{1}$ où $\mathrm{E}^{\mathrm{K}_{1}}$ désigne l'espace des vecteurs de $\mathrm{E}$ invariants par $\mathrm{K}_{1}$ et où $\mathrm{E}_{1}$ n'a aucun sous-quotient isomorphe à $\mathrm{W}$. Quitte à remplacer $\mathrm{K}$ par $\mathrm{K} / \mathrm{K}_{1}$ et $\mathrm{E}$ par $\mathrm{E}^{\mathrm{K}_{1}}$, on peut supposer que $\mathrm{K}$ est un groupe fini.

L'algèbre de groupe $\mathrm{A}=\mathrm{R}[\mathrm{K}]$ est de dimension finie sur $\mathrm{R}$, en particulier elle est artinienne. On note $\mathfrak{r}$ son radical. D'après le corollaire du $\S 10, n^{\circ} 2$ de $[\mathbf{1}]$, pour tout $A$-module $M$, le radical de $\mathrm{M}$ (c'est-à-dire le plus petit sous-module $\mathrm{N}$ tel que $\mathrm{M} / \mathrm{N}$ soit semi-simple) est égal à $\mathfrak{r M}$. On note $\mathrm{M}[\mathfrak{r}]$ le plus grand sous-module semi-simple de $\mathrm{M}$.

Lemme 2.18. - Soit $\mathrm{M}$ un A-module. Alors $\mathfrak{r M}^{*}$ est égal à l'orthogonal de $\mathrm{M}[\mathfrak{r}]$ dans $\mathrm{M}^{*}$.

Démonstration. - D'abord, la restriction des formes linéaires de $\mathrm{M}$ à $\mathrm{M}[\mathfrak{r}]$ induit un isomorphisme de $M^{*} / \mathrm{M}[\mathfrak{r}]^{\circ}$ sur $\mathrm{M}[\mathfrak{r}]^{*}$. Ce dernier est semi-simple, ce qui implique que $\mathfrak{r} \mathrm{M}^{*} \subseteq \mathrm{M}[\mathfrak{r}]^{\circ}$. En outre, si M est de dimension finie sur R, cette inclusion est une égalité.

Écrivons $\mathrm{M}$ comme la réunion de sous-modules $\mathrm{M}_{i}$ de dimension finie sur $\mathrm{R}$, pour $i \in \mathrm{I}$. Alors $\mathrm{M}^{*}$ est la limite projective des $\mathrm{M}_{i}^{*}\left(\mathrm{si} \mathrm{M}_{i} \subseteq \mathrm{M}_{j}\right.$, on a un morphisme surjectif de $\mathrm{M}_{j}^{*}$ dans $\mathrm{M}_{i}^{*}$ défini par restriction des formes linéaires) et $\mathrm{M}[\mathfrak{r}]^{\circ}$ est la limite projective des $\mathrm{M}_{i}[\mathfrak{r}]^{\circ}$. Pour tout $i \in \mathrm{I}$, on a le diagramme commutatif suivant :

$$
\begin{array}{ccc}
\mathfrak{r} M^{*} & \subseteq & \mathrm{M}[\mathfrak{r}]^{\circ} \\
\downarrow & & \downarrow \\
\mathfrak{r} M_{i}^{*} & = & M_{i}[\mathfrak{r}]^{\circ}
\end{array}
$$

et il s'agit donc de prouver que $\mathfrak{r} \mathrm{M}^{*}$ est la limite projective des $\mathfrak{r} \mathrm{M}_{i}^{*}$. Plus précisément, on a un homomorphisme injectif $\xi$ de $\mathfrak{r} M^{*}$ vers la limite projective des $\mathfrak{r} M_{i}^{*}$, et il s'agit de montrer que $\xi$ est surjectif.

Choisissons une base $\left(a_{1}, \ldots, a_{k}\right)$ de $\mathfrak{r}$ comme espace vectoriel sur R. Pour chaque $i \in \mathrm{I}$, on a une application linéaire $f_{i}$ de $\left(\mathrm{M}_{i}^{*}\right)^{k}$ dans $\mathrm{M}_{i}^{*}$ définie par :

$$
f_{i}\left(m_{1}, \ldots, m_{k}\right)=a_{1} m_{1}+\cdots+a_{k} m_{k},
$$

d'image $\mathfrak{r M}_{i}^{*}$. De façon analogue, on a une application linéaire $f$ de $\left(\mathrm{M}^{*}\right)^{k}$ dans $\mathrm{M}^{*}$ dont l'image est $\mathfrak{r M} \mathrm{M}^{*}$. Soit $x \in \mathrm{M}^{*}$ et soit $x_{i}$ son image dans $\mathrm{M}_{i}^{*}$. On suppose que $x_{i} \in \mathfrak{r} \mathrm{M}_{i}^{*}$ pour chaque $i \in \mathrm{I}$. Alors $f_{i}^{-1}\left(x_{i}\right)$ est un sous-espace affine non vide de $\left(\mathrm{M}_{i}^{*}\right)^{k}$ de direction $\operatorname{Ker}\left(f_{i}\right)$. D'après le théorème 1 de [2], chapitre III, $\S 7, \mathrm{n}^{\circ} 4$, la limite projective des $f_{i}^{-1}\left(x_{i}\right)$ est non vide, c'est-à-dire que l'équation $f(y)=x$ admet une solution dans $\left(\mathrm{M}^{*}\right)^{k}$.

Si l'on applique le lemme à $\mathrm{M}=\mathrm{E}$, on obtient un isomorphisme de $\mathrm{E}^{*} / \mathfrak{r} \mathrm{E}^{*}=\mathrm{E}^{*} / \mathrm{E}[\mathrm{r}]^{\circ}$ dans $\mathrm{E}[\mathfrak{r}]^{*}$. Comme $\mathrm{W}$ n'apparait pas dans $\mathrm{E}[\mathfrak{r}]$, on en déduit que $\mathrm{W}^{\vee}$ n'apparaît pas comme quotient de $\mathrm{E}^{*}$.

\section{§3. Modules d'entrelacement}

3.1. - On suppose maintenant que $\mathrm{G}$ est un groupe localement profini et que $\mathrm{K}$ est un sous-groupe ouvert et compact de G. On fixe comme précédemment une représentation lisse absolument irréductible $(\varrho, \mathrm{W})$ de $\mathrm{K}$. On note $\mathcal{H}=\mathcal{H}(\mathrm{G}, \mathrm{K}, \mathrm{W})$ l'algèbre d'entrelacement - ou 
algèbre de Hecke - de $\varrho$ dans $\mathrm{G}$ : on la voit comme formée des fonctions $\mathrm{T}$ de $\mathrm{G}$ dans $\operatorname{End}_{\mathrm{R}}(\mathrm{W})$, à support compact, telles que :

$$
\mathrm{T}(h g k)=\varrho(h) \circ \mathrm{T}(g) \circ \varrho(k)
$$

pour $g \in \mathrm{G}$ et $h, k \in \mathrm{K}$. Cette formule signifie que $\mathrm{T}(g)$ entrelace $\varrho$ avec $\varrho^{g}$. La loi de composition est la loi de convolution :

$$
\mathrm{T} * \mathrm{~S}(g)=\sum_{\mathrm{K} \backslash \mathrm{G}} \mathrm{T}\left(g x^{-1}\right) \circ \mathrm{S}(x)
$$

où la somme porte sur un système quelconque de représentants $x$ de $\mathrm{K} \backslash \mathrm{G}$ dans $\mathrm{G}$.

On note $\mathcal{H}^{\vee}=\mathcal{H}\left(\mathrm{G}, \mathrm{K}, \mathrm{W}^{\vee}\right)$ l'algèbre de Hecke de $\varrho^{\vee}$ dans $\mathrm{G}$. L'application qui à $\mathrm{T}$ associe $\mathrm{T}^{\vee}: g \mapsto \mathrm{T}\left(g^{-1}\right)^{\vee}$ (où $\mathrm{T}\left(g^{-1}\right)^{\vee}$ désigne l'endomorphisme transposé de $\mathrm{T}\left(g^{-1}\right)$ ) est, comme on le vérifie aussitôt, un anti-isomorphisme de R-algèbres de $\mathcal{H}$ sur $\mathcal{H}^{\vee}$.

3.2. - Soit $(\pi, \mathrm{V})$ une représentation lisse de G. L'espace d'entrelacement $\mathrm{Hom}_{\mathrm{K}}(\mathrm{W}, \mathrm{V})$ est alors un $\mathcal{H}$-module à droite : pour $\phi \in \operatorname{Hom}_{\mathrm{K}}(\mathrm{W}, \mathrm{V})$ et $\mathrm{T} \in \mathcal{H}$, on a :

$$
\phi \mathrm{T}=\sum_{\mathrm{K} \backslash \mathrm{G}} \pi\left(x^{-1}\right) \circ \phi \circ \mathrm{T}(x),
$$

la somme portant sur un système quelconque de représentants $x$ de $\mathrm{K} \backslash \mathrm{G}$ dans $\mathrm{G}$. De la même façon, $\operatorname{Hom}_{K}\left(\mathrm{~W}^{\vee}, \mathrm{V}^{\vee}\right)$ est un $\mathcal{H}^{\vee}$-module à droite. C'est également, via l'anti-isomorphisme $\mathrm{T} \mapsto \mathrm{T}^{\vee}$, un $\mathcal{H}$-module à gauche, et il est naturel de se demander si l'accouplement :

$$
\operatorname{Hom}_{\mathrm{K}}(\mathrm{W}, \mathrm{V}) \times \operatorname{Hom}_{\mathrm{K}}\left(\mathrm{W}^{\vee}, \mathrm{V}^{\vee}\right) \rightarrow \mathrm{R}
$$

est hermitien, au sens où :

$$
\langle\phi \mathrm{T}, \psi\rangle=\langle\phi, \mathrm{T} \psi\rangle
$$

pour tous $\phi \in \operatorname{Hom}_{\mathrm{K}}(\mathrm{W}, \mathrm{V}), \psi \in \operatorname{Hom}_{\mathrm{K}}\left(\mathrm{W}^{\vee}, \mathrm{V}^{\vee}\right)$ et $\mathrm{T} \in \mathcal{H}$, où $\mathrm{T} \psi$ désigne bien sûr $\psi \mathrm{T}^{\vee}$.

Il sera commode d'utiliser la remarque 2.2 et de considérer plutôt l'accouplement :

$$
\operatorname{Hom}_{\mathrm{K}}(\mathrm{W}, \mathrm{V}) \times \operatorname{Hom}_{\mathrm{K}}(\mathrm{V}, \mathrm{W}) \rightarrow \mathrm{R} .
$$

L'action à gauche de $\mathcal{H}$ sur $\operatorname{Hom}_{\mathrm{K}}(\mathrm{V}, \mathrm{W})$ est alors donnée par :

$$
\mathrm{T} \psi=\sum_{\mathrm{K} \backslash \mathrm{G}} \mathrm{T}\left(x^{-1}\right) \circ \psi \circ \pi(x)
$$

pour $\psi \in \operatorname{Hom}_{\mathrm{K}}(\mathrm{V}, \mathrm{W})$ et $\mathrm{T} \in \mathcal{H}$, la somme portant sur un système quelconque de représentants de $\mathrm{K} \backslash \mathrm{G}$ dans $\mathrm{G}$.

Remarque 3.1. - Comme l'induction lisse de $\mathrm{K}$ à $\mathrm{G}$ est adjointe à droite de la restriction de $\mathrm{G}$ à $\mathrm{K}$, l'espace $\operatorname{Hom}_{\mathrm{K}}(\mathrm{V}, \mathrm{W})$ est naturellement un module à gauche sur l'algèbre des endomorphismes de l'induite lisse $\operatorname{Ind}_{\mathrm{K}}^{\mathrm{G}}(\varrho)$, notée $\mathcal{A}$. Le produit de convolution :

$$
\mathrm{T} * f: g \mapsto \sum_{\mathrm{K} \backslash \mathrm{G}} \mathrm{T}\left(x^{-1}\right)(f(x g))
$$

pour $\mathrm{T} \in \mathcal{H}, f \in \operatorname{Ind}_{\mathrm{K}}^{\mathrm{G}}(\varrho)$ et $g \in \mathrm{G}$, la somme portant sur un système quelconque de représentants de $\mathrm{K} \backslash \mathrm{G}$ dans $\mathrm{G}$, fait de cette induite lisse un module à gauche sur $\mathcal{H}$ (on vérifie immédiatement que $(\mathrm{T} * \mathrm{~S}) * f=\mathrm{T} *(\mathrm{~S} * f)$ pour tous $\mathrm{T}, \mathrm{S} \in \mathcal{H})$. Ceci définit un morphisme de R-algèbres $i$ de $\mathcal{H}$ dans $\mathcal{A}$. La restriction du $\mathcal{A}$-module $\operatorname{Hom}_{\mathrm{K}}(\mathrm{V}, \mathrm{W})$ à $\mathcal{H}$ induite par le morphisme $i$ coïncide 
avec la structure de $\mathcal{H}$-module à gauche sur $\operatorname{Hom}_{\mathrm{K}}(\mathrm{V}, \mathrm{W})$ définie par (3.3). En effet, l'action de $\mathcal{A}$ sur $\operatorname{Hom}_{\mathrm{K}}(\mathrm{V}, \mathrm{W})$ est donnée par :

$$
a \psi: v \mapsto a(\widetilde{\psi}(v))(1),
$$

pour $a \in \mathcal{A}, \psi \in \operatorname{Hom}_{\mathrm{K}}(\mathrm{V}, \mathrm{W}), v \in \mathrm{V}$, où $\widetilde{\psi}(v)$ est l'élément de $\operatorname{Ind}_{\mathrm{K}}^{\mathrm{G}}(\varrho)$ défini par $g \mapsto \psi(\pi(g) v)$. Par conséquent, pour $\mathrm{T} \in \mathcal{H}$, on a :

$$
i(\mathrm{~T}) \psi: v \mapsto i(\mathrm{~T})(\tilde{\psi}(v))(1)=(\mathrm{T} * \tilde{\psi}(v))(1)=\sum_{\mathrm{K} \backslash \mathrm{G}} \mathrm{T}\left(x^{-1}\right)(\psi(\pi(x) v)
$$

c'est-à-dire que $i(\mathrm{~T}) \psi$ est égal à $\mathrm{T} \psi$ d'après la formule (3.3).

Il s'agit donc de comparer $\psi \circ(\phi \mathrm{T})$ et $(\mathrm{T} \psi) \circ \phi$ pour $\phi \in \operatorname{Hom}_{\mathrm{K}}(\mathrm{W}, \mathrm{V})$ et $\psi \in \operatorname{Hom}_{\mathrm{K}}(\mathrm{V}, \mathrm{W})$. Fixons de tels $\phi$ et $\psi$.

Lemme 3.2. - (1) Soit $g \in \mathrm{G}$. L'opérateur :

$$
\mathrm{E}(\phi, \psi, g)=\psi \circ \pi(g) \circ \phi .
$$

entrelace $\varrho$ avec $\varrho^{g}$.

(2) L'application $\mathrm{E}: g \mapsto \mathrm{E}(\phi, \psi, g)$ de $\mathrm{G}$ dans $\operatorname{End}_{\mathrm{R}}(\mathrm{W})$ vérifie :

$$
\mathrm{E}\left(k g k^{\prime}\right)=\varrho(k) \circ \mathrm{E}(g) \circ \varrho\left(k^{\prime}\right)
$$

pour tous $k, k^{\prime} \in \mathrm{K}$ et $g \in \mathrm{G}$.

Remarque 3.3. - La restriction de $\mathrm{E}$ à chaque double classe $\mathrm{K} g \mathrm{~K}$ de $\mathrm{G}$ est dans $\mathcal{H}$.

Démonstration. - On pose $\mathrm{E}(g)=\mathrm{E}(\phi, \psi, g)$. Pour tout $k \in \mathrm{K} \cap g^{-1} \mathrm{~K} g$, on a :

$$
\begin{aligned}
\varrho\left(g k g^{-1}\right) \circ \mathrm{E}(g) & =\psi \circ \pi\left(g k g^{-1}\right) \circ \pi(g) \circ \phi \\
& =\psi \circ \pi(g) \circ \pi(k) \circ \phi \\
& =\mathrm{E}(g) \circ \varrho(k),
\end{aligned}
$$

ce qui prouve le point 1 . Le point 2 se déduit d'un calcul immédiat.

Soit $\mathrm{T} \in \mathcal{H}$. On a les formules :

$$
\begin{aligned}
\psi \circ \phi \mathrm{T} & =\sum_{\mathrm{K} \backslash \mathrm{G}} \mathrm{E}\left(\phi, \psi, x^{-1}\right) \circ \mathrm{T}(x), \\
\mathrm{T} \psi \circ \phi & =\sum_{\mathrm{K} \backslash \mathrm{G}} \mathrm{T}\left(x^{-1}\right) \circ \mathrm{E}(\phi, \psi, x)
\end{aligned}
$$

dans $\operatorname{End}_{K}(W)$, la somme portant sur un système quelconque de représentants de $K \backslash G$ dans G. Ces deux quantités sont des homothéties de W, c'est-à-dire des endomorphismes de W de la forme $\lambda \cdot \operatorname{id}_{\mathrm{W}}$ avec $\lambda \in \mathrm{R}^{\times}$.

Pour comparer ces deux quantités, il suffit de le faire pour T supporté par une seule double classe $\mathrm{K} g \mathrm{~K}$. Dans ce cas, les formules ci-dessus deviennent :

$$
\begin{aligned}
\psi \circ \phi \mathrm{T} & =\sum_{\left(\mathrm{K} \cap \mathrm{K}^{g}\right) \backslash \mathrm{K}} \varrho\left(x^{-1}\right) \circ \mathrm{E}\left(\phi, \psi, g^{-1}\right) \circ \mathrm{T}(g) \circ \varrho(x), \\
\mathrm{T} \psi \circ \phi & =\sum_{\left(\mathrm{K} \cap \mathrm{K}^{-1}\right) \backslash \mathrm{K}} \varrho\left(x^{-1}\right) \circ \mathrm{T}(g) \circ \mathrm{E}\left(\phi, \psi, g^{-1}\right) \circ \varrho(x)
\end{aligned}
$$


dans $\operatorname{End}_{\mathrm{K}}(\mathrm{W})$, où l'on a noté $\mathrm{K}^{g}=g^{-1} \mathrm{~K} g$ pour $g \in \mathrm{G}$ et où les sommes portent sur des systèmes de représentants des ensembles-quotients indiqués.

Théorème 3.4. - Si la dimension de $\mathrm{W}$ est première à l'exposant caractéristique de $\mathrm{R}$, alors l'accouplement (3.1) est hermitien quelle que soit la représentation lisse $(\pi, \mathrm{V})$ de $\mathrm{G}$.

Démonstration. - En effet, les termes des deux sommes (3.5) et (3.6) ont la même trace, et les deux sommes ont le même nombre de termes, à savoir le nombre de classes de $\mathrm{K}$ dans $\mathrm{K} g \mathrm{~K}$, à droite ou à gauche. Comme les deux sommes sont des homothéties de $\mathrm{W}$, et comme la dimension de $\mathrm{W}$ est première à l'exposant caractéristique de $\mathrm{R}$, le résultat s'ensuit.

3.3. - Le théorème précédent appelle un certain nombre de remarques.

(1) Nous n'avons pas fait d'hypothèse sur la non-dégénérescence de l'accouplement, qui ne semble guère reliée avec le fait qu'il soit hermitien.

(2) Nous ne donnons pas d'exemple où l'accouplement ne soit pas hermitien. Si l'on revient au lemme 3.2, il paraît peu vraisemblable qu'on ait toujours $\mathrm{E}\left(g^{-1}\right) \circ \mathrm{T}(g)=\mathrm{T}(g) \circ \mathrm{E}\left(g^{-1}\right)$. Cependant, une situation fréquente en théorie des types $[\mathbf{3}, \mathbf{6}]$ est celle où :

$$
\operatorname{dim} \operatorname{Hom}_{\mathrm{K} \cap \mathrm{K}^{g}}\left(\varrho, \varrho^{g}\right) \leqslant 1
$$

pour tout $g \in \mathrm{G}$. Alors $\mathrm{E}\left(g^{-1}\right)$ et $\mathrm{T}(g)$ sont pris chacun dans un espace vectoriel de rang 1 , et on peut penser qu'il y a plus de chances qu'ils commutent : voir la section 4 .

(3) Supposons que $(\pi, \mathrm{V})$ est telle que $\mathrm{V}(\varrho)$ et $\mathrm{V}_{\varrho}$ soient irréductibles. Alors ou bien l'application $\mathrm{V}(\varrho) \rightarrow \mathrm{V}_{\varrho}$ est nulle et l'accouplement est nul également, donc hermitien. Ou bien cette application est bijective, et l'accouplement est non dégénéré ; alors $\operatorname{Hom}_{K}(\mathrm{~W}, \mathrm{~V})$ et $\operatorname{Hom}_{\mathrm{K}}(\mathrm{V}, \mathrm{W})$ sont de dimension 1, et pour prouver que l'accouplement est hermitien, il suffit de prouver que les quantités (3.5) et (3.6) sont égales quand $\phi$ et $\psi$ sont des bases de ces espaces, telles qu'on ait $\psi \circ \phi=i d_{W}$. En ce cas, il y a des caractères $\mathrm{A}$ et $\mathrm{B}$ de l'algèbre $\mathcal{H}$ tels que :

$$
\psi \circ \phi \mathrm{T}=\mathrm{A}(\mathrm{T}) \cdot \mathrm{id}{ }_{\mathrm{W}}, \quad \mathrm{T} \psi \circ \phi=\mathrm{B}(\mathrm{T}) \cdot \mathrm{id}_{\mathrm{W}},
$$

pour tout $\mathrm{T} \in \mathcal{H}$, et il suffit de prouver $\mathrm{A}(\mathrm{T})=\mathrm{B}(\mathrm{T})$ pour $\mathrm{T}$ parcourant un système générateur de cette algèbre.

3.4. - Notons $\mathcal{C}$ l'espace des fonctions de $\mathrm{G}$ dans $\operatorname{End}_{\mathrm{R}}(\mathrm{W})$ vérifiant la condition (3.4). Identifions $\mathcal{H}$ au sous-espace de $\mathcal{C}$ formé des fonctions à support compact. Alors $\mathcal{C}$ est naturellement un module à droite et à gauche sur $\mathcal{H}:$ pour $f \in \mathcal{C}$ et $\mathrm{T} \in \mathcal{H}$, on a :

$$
\begin{aligned}
f * \mathrm{~T}: g & \mapsto \sum_{\mathrm{K} \backslash \mathrm{G}} f\left(g x^{-1}\right) \circ \mathrm{T}(x), \\
\mathrm{T} * f: g & \mapsto \sum_{\mathrm{K} \backslash \mathrm{G}} \mathrm{T}\left(g x^{-1}\right) \circ f(x),
\end{aligned}
$$

la somme portant sur un système quelconque de représentants de $K \backslash G$ dans $G$ (on vérifie immédiatement que l'on a $f *(\mathrm{~T} * \mathrm{~S})=(f * \mathrm{~T}) * \mathrm{~S}$ et $(\mathrm{T} * \mathrm{~S}) * f=\mathrm{T} *(\mathrm{~S} * f)$ pour tout $f \in \mathcal{C}$ et tous $\mathrm{T}, \mathrm{S} \in \mathcal{H})$.

Lemme 3.5. - Soit $\mathrm{T} \in \mathcal{H}$ supporté par une seule double classe $\mathrm{K} g \mathrm{~K}$, et soient $f, f^{\prime} \in \mathcal{C}$. On suppose que $f\left(g^{-1}\right)=f^{\prime}\left(g^{-1}\right)$. Alors $f * \mathrm{~T}(1)=f^{\prime} * \mathrm{~T}(1)$ et $\mathrm{T} * f(1)=\mathrm{T} * f^{\prime}(1)$. 
Démonstration. - De façon analogue à (3.5) et (3.6), on a :

$$
\begin{aligned}
& f * \mathrm{~T}(1)=\sum_{\left(\mathrm{K} \cap \mathrm{K}^{g}\right) \backslash \mathrm{K}} \varrho\left(x^{-1}\right) \circ f\left(g^{-1}\right) \circ \mathrm{T}(g) \circ \varrho(x), \\
& \mathrm{T} * f(1)=\sum_{\left(\mathrm{K} \cap \mathrm{K}^{g^{-1}}\right) \backslash \mathrm{K}} \varrho\left(x^{-1}\right) \circ \mathrm{T}(g) \circ f\left(g^{-1}\right) \circ \varrho(x),
\end{aligned}
$$

ce qui prouve le résultat.

On forme les hypothèses suivantes.

Hypothèse 1. - Pour tout $g \in \mathrm{G}$, l'espace d'entrelacement $\operatorname{Hom}_{\mathrm{K}_{\cap} \mathrm{K}^{g}}\left(\varrho, \varrho^{g}\right)$ est de dimension au plus 1.

Hypothèse 2. - Tout élément non nul de $\mathcal{H}$ supporté par une seule double classe est inversible.

Ces hypothèses sont vérifiées fréquemment en théorie des types ([6], lemme 2.19 et corollaire $2.23)$.

Proposition 3.6. - Sous les hypothèses 1 et 2, l'accouplement (3.2) est hermitien.

Démonstration. - Soient $\phi \in \operatorname{Hom}_{\mathrm{K}}(\mathrm{W}, \mathrm{V}), \psi \in \operatorname{Hom}_{\mathrm{K}}(\mathrm{V}, \mathrm{W})$ et $\mathrm{T} \in \mathcal{H}$. On suppose que $\mathrm{T}$ est supporté par une seule double classe $\mathrm{K} g \mathrm{~K}$. D'après l'hypothèse 2 , $\mathrm{T}$ est inversible dans $\mathcal{H}$. Ainsi $\mathrm{T}^{-1}\left(g^{-1}\right)$ entrelace $\varrho$ avec $\varrho^{g^{-1}}$.

Lemme 3.7. - $\mathrm{T}^{-1}\left(g^{-1}\right)$ est non nul.

Démonstration. - Par définition du produit de convolution, on a :

$$
\operatorname{id} \mathrm{W}=\mathrm{T}^{-1} * \mathrm{~T}(1)=\sum_{\left(\mathrm{K} \cap \mathrm{K}^{g}\right) \backslash \mathrm{K}} \varrho\left(x^{-1}\right) \circ \mathrm{T}^{-1}\left(g^{-1}\right) \circ \mathrm{T}(g) \circ \varrho(x),
$$

où la somme porte sur un système quelconque de représentants de $\mathrm{K} \cap \mathrm{K}^{g}$ dans $\mathrm{K}$. Par conséquent, $\mathrm{T}^{-1}\left(g^{-1}\right)$ n'est pas nul.

D'après le lemme 3.2, l'opérateur $\mathrm{E}\left(\phi, \psi, g^{-1}\right)$ entrelace $\varrho$ avec $\varrho^{g^{-1}}$. D'après l'hypothèse 1 , il $\mathrm{y}$ a donc un scalaire $z \in \mathrm{R}$ tel que :

$$
\mathrm{E}\left(\phi, \psi, g^{-1}\right)=z \mathrm{~T}^{-1}\left(g^{-1}\right) .
$$

Si l'on applique le lemme 3.5 avec $f=\mathrm{E}$ et $f^{\prime}=z \mathrm{~T}^{-1}$, on a :

$$
\mathrm{E} * \mathrm{~T}(1)=z \mathrm{~T}^{-1} * \mathrm{~T}(1)=z \mathrm{~T} * \mathrm{~T}^{-1}(1)=\mathrm{T} * \mathrm{E}(1),
$$

c'est-à-dire que (3.5) et (3.6) coïncident.

Remarque 3.8. - L'hypothèse 2 pourrait être remplacée par l'hypothèse plus faible suivante : pour tout $g \in \mathrm{G}$ et tout $\mathrm{T} \in \mathcal{H}$ de support $\mathrm{K} g \mathrm{~K}$, il y a une fonction $f \in \mathcal{C}$ telle que $f\left(g^{-1}\right) \neq 0$ et $f * \mathrm{~T}(1)=\mathrm{T} * f(1)$.

Si l'on ajoute aux considérations ci-dessus celles des paragraphes 2.5 et 2.6 , on a le théorème suivant. 
Théorème 3.9. - Supposons que les hypothèses 1 et 2 sont vérifiées et que l'induite compacte $\operatorname{ind}_{\mathrm{K}}^{\mathrm{G}}(\varrho)$ est fortement $\mathrm{W}$-semi-simple. Alors pour toute représentation lisse $\mathrm{V}$ de $\mathrm{G}$ qui est sousquotient d'une représentation engendrée par son composant isotypique de type $\varrho$, les $\mathcal{H}$-modules $\operatorname{Hom}_{\mathrm{K}}(\mathrm{V}, \mathrm{W})$ et $\operatorname{Hom}_{\mathrm{K}}(\mathrm{W}, \mathrm{V})^{*}$ sont isomorphes.

Démonstration. - Une telle représentation V est sous-quotient d'une somme directe arbitraire de copies de $\operatorname{ind}_{\mathrm{K}}^{\mathrm{G}}(\varrho)$. Le résultat est alors une conséquence des propositions 2.6, 2.12 et 3.6.

Il se peut que $\operatorname{Hom}_{K}(\mathrm{~V}, \mathrm{~W})$ et $\operatorname{Hom}_{\mathrm{K}}(\mathrm{W}, \mathrm{V})^{*}$ soient des $\mathcal{H}$-modules à gauche isomorphes sans que cet isomorphisme ne provienne de l'accouplement (3.2).

Exemple 3.10. - Supposons que R est de caractéristique $\ell>0$, et supposons que $\mathrm{G}=\mathrm{K}$ est un groupe cyclique d'ordre $\ell$. Soit $(\pi, \mathrm{V})$ une auto-extension non triviale du caractère trivial de G et soit $(\varrho, \mathrm{W})$ le caractère trivial de K. L'accouplement (3.2) est nul ; pourtant, les $\mathcal{H}$-modules $\operatorname{Hom}_{\mathrm{K}}(\mathrm{V}, \mathrm{W})$ et $\operatorname{Hom}_{\mathrm{K}}(\mathrm{W}, \mathrm{V})^{*}$ sont tous deux isomorphes au caractère trivial de $\mathcal{H}$.

3.5. - Revenons à la notion de $\mathrm{W}$-semi-simplicité définie au paragraphe 2.3 .

Proposition 3.11. - Supposons que les hypothèses 1 et 2 sont vérifiées. Alors l'induite compacte $\operatorname{ind}_{\mathrm{K}}^{\mathrm{G}}(\varrho)$ est $\mathrm{W}$-semi-simple.

Démonstration. - D'après la formule de Mackey et la remarque 2.10, pour que l'induite compacte $\operatorname{ind}_{\mathrm{K}}^{\mathrm{G}}(\varrho)$ soit W-semi-simple, il faut et il suffit que, pour chaque $g \in \mathrm{G}$, l'espace :

$$
\mathrm{I}(g)=\operatorname{ind}_{\mathrm{K} \cap \mathrm{K}^{g}}^{\mathrm{K}}\left(\varrho^{g}\right)
$$

le soit. Étant donné $g \in \mathrm{G}$, l'hypothèse 2 implique (voir le lemme 3.7) que :

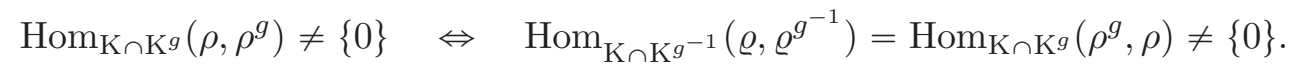

En d'autres termes, $\operatorname{Hom}_{K}(\mathrm{~W}, \mathrm{I}(g))$ est nul si et seulement si $\operatorname{Hom}_{\mathrm{K}}(\mathrm{I}(g), \mathrm{W})$ est nul, auquel cas $\mathrm{I}(g)$ est W-semi-simple.

Dans le cas où ces espaces sont non nuls, ce que nous supposons maintenant jusqu'à la fin de la preuve, ils sont de dimension 1 d'après l'hypothèse 1 . Fixons un élément non nul $\mathrm{T} \in \mathcal{H}$ supporté par la double classe $\mathrm{K} g \mathrm{~K}$. Alors $\mathrm{T}(g) \in \operatorname{Hom}_{\mathrm{K}_{n} \mathrm{~K}^{g}}\left(\varrho, \varrho^{g}\right)$ est un opérateur d'entrelacement non nul et si l'on note $k \cdot w$ le vecteur $\varrho(k)(w)$ pour $w \in \mathrm{W}$ et $k \in \mathrm{K}$, l'application $\alpha \in \operatorname{Hom}_{\mathrm{K}}(\mathrm{W}, \mathrm{I}(g))$ définie par :

$$
\alpha(w)(k)=\mathrm{T}(g)(k \cdot w)
$$

pour $w \in \mathrm{W}$ et $k \in \mathrm{K}$ est un générateur de $\operatorname{Hom}_{\mathrm{K}}(\mathrm{W}, \mathrm{I}(g))$. Parallèlement, d'après le lemme 3.7 à nouveau, $\mathrm{T}^{-1}\left(g^{-1}\right) \in \operatorname{Hom}_{\mathrm{K} \cap \mathrm{K}^{g}}\left(\varrho^{g}, \varrho\right)$ est un opérateur d'entrelacement non nul. Si pour tout $w \in \mathrm{W}$ et tout $k \in \mathrm{K}$ on note $[k, w]$ l'élément de $\mathrm{I}(g)$ de support $\left(\mathrm{K} \cap \mathrm{K}^{g}\right) k$ et prenant en $k$ la valeur $w$, alors l'application $\beta \in \operatorname{Hom}_{\mathrm{K}}(\mathrm{I}(g), \mathrm{W})$ définie par :

$$
\beta([k, w])=k^{-1} \cdot \mathrm{T}^{-1}\left(g^{-1}\right)(w)
$$

pour $w \in \mathrm{W}$ et $k \in \mathrm{K}$ est un générateur de $\operatorname{Hom}_{\mathrm{K}}(\mathrm{I}(g), \mathrm{W})$. Ainsi $\mathrm{I}(g)$ est W-semi-simple si et seulement si $\beta \circ \alpha$ est un opérateur non nul de $\operatorname{End}_{\mathrm{K}}(\mathrm{W})$. Pour tout $w \in \mathrm{W}$, on a :

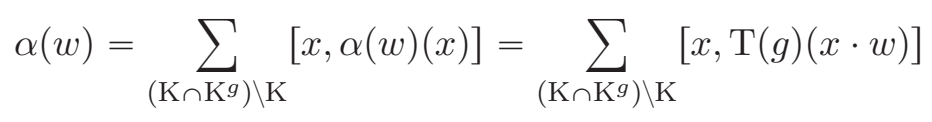


où $x$ décrit un système quelconque de représentants de $\left(\mathrm{K} \cap \mathrm{K}^{g}\right) \backslash \mathrm{K}$ dans $\mathrm{K}$, donc :

$$
\beta(\alpha(w))=\sum_{\left(\mathrm{K} \cap \mathrm{K}^{g}\right) \backslash \mathrm{K}} x^{-1} \cdot \mathrm{T}^{-1}\left(g^{-1}\right)(\mathrm{T}(g)(x \cdot w))
$$

c'est-à-dire que :

$$
\beta \circ \alpha=\sum_{\left(\mathrm{K} \cap \mathrm{K}^{g}\right) \backslash \mathrm{K}} \varrho\left(x^{-1}\right) \circ \mathrm{T}^{-1}\left(g^{-1}\right) \circ \mathrm{T}(g) \circ \varrho(x) .
$$

Ainsi, on trouve que $\beta \circ \alpha$ est égal à $\mathrm{T}^{-1} * \mathrm{~T}(1)=\mathrm{id}_{\mathrm{W}}$.

On peut se demander s'il y a des exemples où les hypothèses 1 et 2 sont vérifiées et où l'induite compacte $\operatorname{ind}_{\mathrm{K}}^{\mathrm{G}}(\varrho)$ n'est pas fortement W-semi-simple. Nous ne connaissons pas de tels exemples. En revanche, nous avons le résultat suivant.

Proposition 3.12. - Supposons que $\mathrm{R}$ est de caractéristique $\ell>0$, et supposons que @ est le caractère trivial de $\mathrm{K}$. Les conditions suivantes sont équivalentes.

(1) Pour tout $g \in \mathrm{G}$, l'indice de $\mathrm{K} \cap \mathrm{K}^{g}$ dans $\mathrm{K}$ est premier à $\ell$.

(2) L'induite compacte $\operatorname{ind}_{\mathrm{K}}^{\mathrm{G}}(1)$ est $\mathrm{W}$-semi-simple.

Démonstration. - Remarquons d'abord que le caractère trivial de $\mathrm{K}$ vérifie automatiquement l'hypothèse 1. Plus précisément, et avec les notations de la preuve de la proposition 3.11, l'unique sous-espace de $\mathrm{I}(g)$, pour $g \in \mathrm{G}$, qui soit invariant par K est celui des fonctions constantes, tandis que son unique (à un scalaire près) forme linéaire invariante par K est donnée par la somme des valeurs. Il s'ensuit que $\mathrm{I}(g)$ est $\mathrm{W}$-semi-simple si et seulement si l'indice de $\mathrm{K} \cap \mathrm{K}^{g}$ dans $\mathrm{K}$ est premier à $\ell$.

Voici deux exemples qui illustrent la proposition 3.12.

Exemple 3.13. - Supposons que R est de caractéristique $\ell>0$.

(1) Si K est un pro- $\ell$-groupe, alors $\varrho$ est le caractère trivial de $\mathrm{K}$ et $\operatorname{ind}_{\mathrm{K}}^{\mathrm{G}}(1)$ est W-semi-simple si et seulement si K est normal dans G.

(2) Soit $\mathrm{F}$ un corps localement compact et non archimédien, et soit $\mathrm{G}$ le groupe $\mathrm{GL}_{n}(\mathrm{~F})$ avec $n \geqslant 2$. Supposons que $(\varrho, \mathrm{W})$ est le caractère trivial d'un sous-groupe compact maximal $\mathrm{K} \subseteq \mathrm{G}$. Alors l'induite ind $\mathrm{K}_{\mathrm{K}}^{\mathrm{G}}(1)$ est W-semi-simple si et seulement si le cardinal $q$ du corps résiduel de $\mathrm{F}$ et tous les $1+q+\cdots+q^{k}$ avec $k \in\{1, \ldots, n-1\}$ sont premiers à $\ell$.

3.6. - Pour terminer cette section, on fait le lien entre la $\mathrm{W}$-semi-simplicité forte et la notion de quasi-projectivité introduite dans l'appendice de [10].

Définition 3.14. - Une représentation lisse Q de G est quasi-projective si, pour tout homomorphisme surjectif $f$ de $\mathrm{Q}$ dans une représentation $\mathrm{V}$, l'homomorphisme de $\operatorname{End}_{G}(\mathrm{Q})$-modules de $\operatorname{End}_{\mathrm{G}}(\mathrm{Q})$ dans $\operatorname{Hom}_{\mathrm{G}}(\mathrm{Q}, \mathrm{V})$ défini par $u \mapsto f \circ u$ est surjectif.

Proposition 3.15. - Supposons que l'induite compacte $\operatorname{ind}_{\mathrm{K}}^{\mathrm{G}}(\varrho)$ est fortement $\mathrm{W}$-semi-simple. Alors elle est quasi-projective et de type fini.

Démonstration. - On note $\mathrm{Q}$ l'induite compacte $\operatorname{ind}_{\mathrm{K}}^{\mathrm{G}}(\varrho)$. Soit $f: \mathrm{Q} \rightarrow \mathrm{V}$ un homomorphisme surjectif de représentations de G. D'après la proposition 2.12, V est fortement W-semi-simple 
puisque Q l'est, et $f$ induit un homomorphisme surjectif K-équivariant de $\mathrm{Q}(\varrho)$ dans $\mathrm{V}(\varrho)$. On en déduit un homomorphisme surjectif :

$$
\operatorname{Hom}_{\mathrm{K}}(\mathrm{W}, \mathrm{Q}(\varrho)) \rightarrow \operatorname{Hom}_{\mathrm{K}}(\mathrm{W}, \mathrm{V}(\varrho))
$$

Le membre de droite est égal à $\operatorname{Hom}_{K}(\mathrm{~W}, \mathrm{~V})$, et le membre de gauche est égal à $\operatorname{Hom}_{\mathrm{K}}(\mathrm{W}, \mathrm{Q})$, qui est canoniquement isomorphe à $\operatorname{End}_{G}(Q)$ par réciprocité de Frobenius. L'homomorphisme

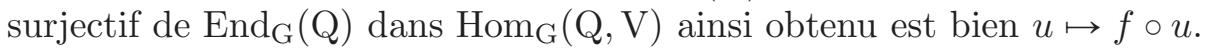

Remarque 3.16. - La proposition 3.15 peut aussi être obtenue en utilisant [11, Lemma 3.1] et la notion de presque-projectivité de [10].

Grâce aux théorèmes 4 et 10 de l'appendice de [10], on déduit de la proposition 3.15 le résultat suivant.

Corollaire 3.17. - Si l'induite compacte $\operatorname{ind}_{\mathrm{K}}^{\mathrm{G}}(\varrho)$ est fortement $\mathrm{W}$-semi-simple, alors le foncteur $\mathrm{V} \mapsto \mathrm{Hom}_{\mathrm{K}}(\mathrm{W}, \mathrm{V})$ possède les propriétés suivantes :

(1) Il induit une bijection entre les classes d'isomorphisme de représentations irréductibles $\mathrm{V}$ de $\mathrm{G}$ telles que $\mathrm{V}(\varrho) \neq\{0\}$ et les classes d'isomorphisme de $\mathcal{H}$-modules à droite simples.

(2) Il est exact sur la sous-catégorie pleine des représentations lisses de G formée des représentations qui sont sous-quotients de représentations engendrées par leur composant isotypique de type $\varrho$.

Remarque 3.18. - La sous-catégorie pleine des représentations lisses de G qui sont sous-quotients de représentations engendrées par leur composante isotypique de type $\varrho$ n'est pas stable par extensions en général : elle ne contient pas la représentation de l'exemple 3.10.

\section{§4. Application aux types simples}

On suppose dans ce paragraphe que $\mathrm{G}$ est le groupe $\mathrm{GL}_{m}(\mathrm{D})$, où $m \geqslant 1$ et où $\mathrm{D}$ est une algèbre à division centrale et de dimension finie sur un corps localement compact et non archimédien $\mathrm{F}$ de caractéristique résiduelle $p$. On suppose que $\mathrm{R}$ est algébriquement clos et de caractéristique différente de $p$, et que $(\mathrm{K}, \varrho, \mathrm{W})$ est un type simple de $\mathrm{G}$ au sens de $[6], \S 2.5$.

D'après [6], lemme 2.19 et corollaire 2.23 , les hypothèses 1 et 2 du paragraphe 3.4 sont satisfaites pour $(\mathrm{K}, \varrho, \mathrm{W})$, et il découle de la preuve de $[\mathbf{6}]$, proposition 4.9 que l'induite compacte $\operatorname{ind}_{\mathrm{K}}^{\mathrm{G}}(\varrho)$ est fortement $\mathrm{W}$-semi-simple. On déduit du théorème 3.9 le résultat suivant.

Théorème 4.1. - Soit $\mathrm{V}$ un sous-quotient d'une représentation de $\mathrm{GL}_{m}(\mathrm{D})$ engendrée par sa composante isotypique de type $\varrho$. Alors les $\mathcal{H}$-modules à gauche $\operatorname{Hom}_{\mathrm{K}}(\mathrm{V}, \mathrm{W})$ et $\operatorname{Hom}_{\mathrm{K}}(\mathrm{W}, \mathrm{V})^{*}$ sont isomorphes.

Ce théorème permet de montrer que la classification de Zelevinski $\mathfrak{m} \mapsto \mathrm{Z}(\mathfrak{m})$ des représentations lisses irréductibles de $\mathrm{GL}_{m}(\mathrm{D})$ à coefficients dans $\mathrm{R}$ en termes de multisegments [7] est compatible au passage à la contragrédiente, c'est-à-dire que si $\mathfrak{m}$ est un multisegment et $\mathfrak{m}^{\vee}$ son multisegment contragrédient, alors $\mathrm{Z}\left(\mathfrak{m}^{\vee}\right)$ est isomorphe à la contragrédiente de $\mathrm{Z}(\mathfrak{m})$. 


\section{Références}

[1] N. Bourbaki, Algèbre, Chapitre 8. Seconde édition, Springer-Verlag, Berlin, 2012.

[2] _ Théorie des ensembles. Springer-Verlag, Berlin, 2006.

[3] C. J. Bushnell \& P. C. Kutzko, "The admissible dual of GL(N) via compact open subgroups", Princeton University Press, Princeton, NJ, 1993.

[4] - "Smooth representations of reductive $p$-adic groups: structure theory via types", Proc. London Math. Soc. (3) 77 (1998), no. 3, p. 582-634.

[5] F. Herzig, "The classification of irreducible admissible mod $p$ representations of a $p$-adic $\mathrm{GL}_{n}$ ", Invent. Math. 186 (2011), n², p. 373-434.

[6] A. Mínguez \& V. SÉcherre, "Types modulo $\ell$ pour les formes intérieures de GL $_{n}$ sur un corps local non archimédien", prépublication, voir http://lmv.math.cnrs.fr/annuaire/vincent-secherre/.

[7] A. Mínguez \& V. SÉcherre, "Représentations lisses modulo $\ell$ de $\mathrm{GL}_{m}(\mathrm{D})$ ", prépublication, voir http://lmv.math.cnrs.fr/annuaire/vincent-secherre/.

[8] M. TADić, "Induced representations of $\operatorname{GL}(n, A)$ for $p$-adic division algebras $A$ ", J. Reine Angew. Math. 405 (1990), p. 48-77.

[9] M.-F. VignéRAs, "Représentations $l$-modulaires d'un groupe réductif $p$-adique avec $l \neq p$ ", Progress in Mathematics, vol. 137, Birkhäuser Boston Inc., Boston, MA, 1996.

[10] _ "Induced R-representations of p-adic reductive groups", Selecta Math. (N.S.) 4 (1998), no. 4, p. 549-623. With an appendix by Alberto Arabia.

[11] _ "Irreducible modular representations of a reductive $p$-adic group and simple modules for Hecke algebras", in European Congress of Mathematics, Vol. I (Barcelona, 2000), Progr. Math., vol. 201, Birkhäuser, Basel, 2001, p. 117-133.

[12] A. V. Zelevinsky, "Induced representations of reductive p-adic groups. II. On irreducible representations of GL(n)", Ann. Sci. École Norm. Sup. (4) 13 (1980), no. 2, p. 165-210.

Guy Henniart, Université de Paris-Sud, Laboratoire de Mathématiques d'Orsay, F-91405 Orsay Cedex E-mail : guy.henniart@math.u-psud.fr

Vincent SÉcherre, Université de Versailles St-Quentin-en-Yvelines, Laboratoire de Mathématiques de Versailles, 45 avenue des Etats-Unis, 78035 Versailles cedex, France • E-mail : vincent.secherre@math.uvsq.fr 